\title{
Une société savante liégeoise dans son contexte social et intellectuel : La société royale des sciences de Liège à ses débuts (1835-ca 1860)
}

\author{
Geneviève XHAYET \\ ULiège-CHST \\ UR Traverses / « Ressources-Pouvoir-Idéologies »
}

\section{Introduction}

Voici un an, la Société royale des Sciences de Liège me faisait l'honneur de me recevoir dans ses rangs et me proposait d'ouvrir le présent colloque avec une contribution relative à son histoire. Plutôt qu'un survol du passé de cette société qui aurait fourni une galerie de portraits et de travaux, certes prestigieuse mais peu significative du point de vue historiographique, j'ai préféré axer mon exposé sur un temps fort de la SRSL, ses débuts en l'occurrence.

La fourchette chronologique choisie s'étend de la création de la Société en 1835, jusqu'aux alentours de 1860, en se souvenant que la SRSL a connu une sorte de faux départ en 1835, et n'a pris son essor définitif qu'en 1842. Cette période est celle des débuts de la Belgique indépendante et, dans le bassin liégeois, d'un développement industriel considérable qui fit de cette région le berceau de l'industrialisation sur le continent européen. Sur le plan scientifique, ce temps est celui d'un essor des universités accompagné d'un renouvellement des conceptions tant méthodologiques qu'épistémologiques de leurs activités. L'objectif est de percevoir dans quelles conditions cette association de savants s'est édifiée et a pris ses marques dans le paysage intellectuel local et international, aux lendemains de la création de l'université de Liège, puis de l'indépendance de la Belgique.

Pour aborder ces questions, l'historien dispose principalement d'archives de la Société ellemême : les recueils de ses Mémoires publiés à partir de 1843, les procès-verbaux de séances et rapports annuels du secrétaire général. Ces sources qui n'existent pas pour la totalité de la période envisagée éclairent divers aspects des activités de la SRSL. Mais l'impression qui en ressort parfois est celle d'une vision «lissée » de la vie de la SRSL. Si écho y est fait à certaines difficultés qu'elle dut affronter (notamment par rapport à l'université), ces sources restent très discrètes quant à d'éventuels débats qui auraient pu éclore au sein même de l'association.

Avant d'entrer dans le vif du sujet, il convient en outre de se souvenir que la SRSL n'a pas surgi du néant mais que dans les siècles antérieurs à sa création, à l'étranger comme à Liège, des compagnies savantes comparables avaient existé, dont ont pu s'inspirer ses initiateurs.

\section{Terreau et antécédents}

Sous l'Ancien Régime, des Académies vouées au progrès des sciences et des techniques se sont créées en marge des universités et, sous l'impulsion des pouvoirs princiers, qui en attendaient surcroît de prestige et retombées concrètes. À partir du milieu du XVIIIe siècle, de tels cercles savants se sont multipliés dans le sillage des Lumières, y compris au sein de cours relativement modestes. Ainsi, en 1750, le roi Stanislas Leszczynski fonde la Société royale des Sciences et des Belles-Lettres de Nancy, tandis qu'à Bruxelles, l'impératrice MarieThérèse institue une Académie impériale des Sciences et des Arts, en 1772. Sept ans plus tard, à Liège, deux sociétés voient le jour à l'initiative du prince-évêque François-Charles de Velbruck : la Société littéraire et la Société de l'Émulation. 


\section{La Société d'Émulation et la Société libre des sciences physiques et médicales}

La Société littéraire est un cercle de notables qui, offrant à ses habitués des journaux ainsi que des tables de jeu, tient à la fois du cabinet de lecture et du casino. Jugée peu dangereuse par les pouvoirs qui se succèdent à la tête de la principauté entre 1789 et la période napoléonienne, la Société littéraire traverse ces temps troublés sans avoir à trop en pâtir.

La destinée de la Société d'Émulation est à la fois plus tumultueuse et plus intéressante pour notre propos. Visant la diffusion des sciences, de l'histoire et des Belles-Lettres, l'Émulation attire parmi ses membres des personnalités telles que l'horloger Hubert Sarton, le physicien François Villette ou les médecins Jean-Philippe de Limbourg et Pierre-Michel Ramoux (17521829). Calquée sur le modèle de l'Académie de Nancy, elle s'inscrit dans le droit fil des Lumières et de la modernité. Durant la Révolution liégeoise, cette option la place dans le collimateur des contre-révolutionnaires qui la démantèlent en 1792, lors du retour au pouvoir du prince évêque Hoensbroek.

En 1806, une société visant à l'avancement des Sciences renaît à Liège, à l'initiative de Pierre Michel Ramoux et avec l'appui du pouvoir napoléonien : la société libre des sciences physiques et médicales de Liège. En 1809, cette société fusionne avec l'Émulation, qui a entre-temps été rétablie. Sa devise «Observatione et experientia» (par l'observation et l'expérience) inscrit ce groupement dans la rationalité et le modernisme scientifiques. Bénéficiant lui-même de l'impulsion donnée aux sciences en France, il promeut surtout la médecine et encourage à Liège le développement de cours d'anatomie, en récompensant les élèves les plus méritants.

\section{L'université de Liège}

La société royale des sciences de Liège s’enracine aussi dans le milieu universitaire.

Une première université créée par un décret impérial de mars 1807 précède la fondation de l'institution liégeoise désormais bicentenaire. Ce premier établissement d'enseignement supérieur comprenait deux facultés, une pour les Lettres et une pour les Sciences, incluant la médecine. Survivant à la chute de l'empire, cet enseignement médical constitua ensuite le noyau de la future université, créée par Guillaume Ier des Pays Bas, en 1817. Comme sa jumelle gantoise, la jeune institution liégeoise comptera quatre facultés pour le droit, la médecine, les sciences ainsi qu'une faculté de philosophie et lettres. Particularité liégeoise directement liée à l'industrialisation fulgurante de la vallée de la Meuse : en 1825, une faculté technique est annexée à la faculté des Sciences. Elle forme des ingénieurs civils des mines ou des Arts et manufactures (en l'occurrence des ingénieurs mécaniciens). À quelques modifications près l'État belge conserve cette structure en 1830.

\section{1835-1842 Genèse et structuration}

\section{Naissance de la Société royale des sciences de Liège}

La société royale des sciences de Liège est fondée le 7 mars 1835. Mais en amont, nous ignorons quand, précisément, avait été prise la décision de l'instituer. Les sources nous informent d'une démarche des fondateurs en ce sens auprès du Ministre de l'Intérieur (en 
charge de l'instruction) Barthélemy de Theux le 23 février 1835. Deux réunions sont ensuite actées sous forme de PV, les 2 et 7 mars. Le bureau est alors élu et les douze membres fondateurs : professeurs à la faculté des sciences, officiers supérieurs de l'armée et ingénieurs, sont répartis au sein de deux grandes sections thématiques : celle des sciences d'observation (chimie, sciences naturelles) et celles des sciences dites «de calcul» (mathématiques et sciences appliquées).

Quatre autres réunions ont encore lieu en 1835, après quoi, la société s'éteint durant six ans. Elle renaît le 26 mars 1842, dans une composition quelque peu modifiée en raison du décès de deux de ses fondateurs, le médecin et paléontologue Philippe Schmerling et l'anatomiste Vincent Fohmann. L'obligation pour les membres effectifs de résider en province de Liège entraîne aussi le retrait du groupe de Gaspard-Michel Pagani. Le mathématicien a en effet quitté entre temps l'université de Liège, pour celle de Louvain. À partir de 1842, la SRSL pérennise ses séances, au rythme de cinq à dix par an, ce qui s'avère très inférieur aux intentions manifestées dans les statuts, de deux réunions mensuelles, de novembre à fin juillet de chaque année.

\section{Structuration et légitimation}

Adoptés le 7 mars 1835, des statuts définissent la société et éclairent ses objectifs. Selon les termes du $1^{\mathrm{er}}$ article, la SRSL favorisera «l'avancement et la propagation des sciences physiques et mathématiques et leurs applications ». Les autres articles règlent la marche de l'association, et fixent le contenu de sa future publication périodique. Impérativement constituée de trois parties, celle-ci proposera un ou des mémoires scientifiques de membres de la Société, un état «du développement des sciences », enfin une analyse critique d'ouvrages reçus. En 1842, la devise choisie pour figurer en exergue «nec temere, nec timide », "sans audace, ni timidité », donnera le ton des futurs travaux.

La jeune société se soucie également de se légitimer dans le paysage institutionnel et scientifique.

Elle se profile d'emblée comme une association belge, implantée à Liège. Agréée par le Ministère de l'Intérieur, elle sollicite le soutien du roi ; un geste qui l'inscrit dans la tradition remontant à l'Ancien Régime d'encouragement de la recherche par les souverains. À cela près toutefois qu'en 1835, la nouvelle institution résulte de l'initiative de ses membres qui sont à l'origine aussi du patronage octroyé par Léopold Ier. Cette obtention, tout comme l'adjonction de la qualification «royal[e] » au nom de l'association justifieront dans l'avenir des demandes de subsides auprès du souverain et/ou du gouvernement. Il ne semble pas, qu'en échange des demandes particulières auprès de la Société aient émané du Palais.

Royale, la société s'affiche également «belge », ce qui se traduit, en 1842, par l'insertion du lion héraldique dans son sceau, obtenue du Ministère de l'intérieur. De même, deux ans plus tard, dans la foulée d'un courant historiographique en vogue qui vise à créer une histoire nationale des sciences, la Société place sur le bandeau bordant ses diplômes les noms de quatre gloires scientifiques nationales : deux médecins Rembert Dodoens et Andriaan van de Spiegel, et deux mathématiciens Simon Stévin et René-François de Sluse, issus des anciens Pays-Bas et de la principauté de Liège. 


\section{Ancrages}

Belge, la société ne sera pas moins liégeoise. L'université est un premier point lieu d'enracinement. Si elle n'est pas à proprement parler « universitaire », la SRSL est néanmoins très nettement dans son orbite. Elle est née de l'initiative de quelques-uns de ses professeurs. La liste de ses membres se confond pour une bonne part avec celle des membres de la Faculté des sciences. Outre Schmerling et Fohmann déjà cités, André Dumont, Adolphe Lesoinne, Michel Gloesener figurent parmi ses fondateurs. Au fil du temps, d'autres noms enrichissent cette première liste, tels ceux de Théodore Schwann, Antoine Spring, Jean-Louis Trasenster pour la période qui nous intéresse. Sur un plan plus matériel, dans les années 1840, la Société installe dans les bâtiments universitaires ses archives et sa bibliothèque. À la fin de cette même décennie, la cession d'une partie de celle-ci aux collections universitaires deviendra une pomme de discorde avec la Société. Il en sera question plus loin.

Le monde industriel est un second point d'ancrage. Lors de la refondation de la Société en 1842, l'intérêt pour l'application des sciences et leur implication dans l'évolution du tissu industriel présent dans les statuts de 1835 est réaffirmé avec force, comme on peut le lire dans les pages liminaires du premier recueil de Mémoires :
La volonté des fondateurs est de «créer dans la province de Liège un centre auquel pussent se rallier toutes les personnes qui, dans ce pays si éminemment industriel, ne se contentent pas de la pratique et se livrent à des recherches sur les principes scientifiques qui lui servent de base ».

En filigrane, perce ainsi le projet de servir l'industrie en la dégageant de l'empirisme, encore trop souvent prévalant à cette époque, pour créer de véritables savoirs industriels basés sur la science.

Les deux secteurs les plus présents dans les préoccupations de la SRSL sont le chemin de fer et surtout les houillères. En juin 1842, l'ingénieur Henri Maus intègre ses rangs et deux ans plus tard, y présente son Rapport à l'appui du projet pour l'établissement des machines et mécanismes destinés au service des plans inclinés de Liège. En 1847, les causes physiques de déraillement des wagons dans les courbes retiennent pareillement l'attention de la Société.

L'exploitation minière est abordée sous différents angles : structure des gisements dans les bassins wallons ou étrangers, machinerie pour l'exhaure, dispositifs d'aération des galeries apparaissent régulièrement sous la plume des membres de la Société, ou dans les travaux qui lui sont offerts. Si les aspects technologiques du travail minier suscitent nombre de commentaires, force est de constater qu'en 1846, là comme dans la plupart des milieux aisés de ce temps, l'indifférence à la détresse des mineurs est grande, semble totale. Les rapports sur la condition ouvrière, ainsi que sur le travail des femmes et des enfants dans les mines déposés par le médecin Nicolas Fossion au bureau de la société n'y suscitent en tout cas, aucune réaction.

\section{Ouverture et réseaux}

Comme les sociétés savantes créées antérieurement, la SRSL se dote d'emblée d'un réseau national et international, tant au niveau individuel, par le choix de correspondants, qu'institutionnel, en tissant un réseau d'échanges avec des sociétés belges ou étrangères. Au 
travers de ces réseaux, se dessine, vue de Liège, une géographie du mouvement scientifique occidental.

\section{Correspondants individuels}

Le réseau des correspondants, élus sur parrainage de deux membres effectifs, s'instaure dès 1835 et crôt rapidement. Si le nombre de ces derniers se stabilise rapidement autour de la trentaine, celui des correspondants s'élève à 65 en 1850 et à 87, deux ans plus tard. Ces correspondants n'apparaissent qu'épisodiquement dans nos sources : lors de leur candidature ou lors de la présentation d'un de leurs travaux. Sans surprise, la politique de recrutement des correspondants consiste à attirer dans les rangs de la SRSL le gratin de la science : membres des universités belges et étrangères, hauts officiers de l'armée ou de l'appareil d'état. Cet objectif fut-il toujours atteint? La SRSL essuya-t-elle des refus ? Des démarches auprès de savants restèrent-elles inabouties? C'est peu probable, mais nos sources ne fournissent aucune certitude à cet égard. Il est en revanche manifeste que la SRSL put s'enorgueillir de correspondants restés célèbres dans l'histoire de la science en Belgique. On citera JeanBaptiste d'Omalius d'Halloy, Adolphe Quételet, Théodore Schwann (alors à Louvain) et Pierre-Joseph Van Beneden. Sur le plan international, des noms aussi se détachent, tels ceux du chimiste allemand Justus von Liebig ou de Charles Lyell, président de la société géologique de Londres.

\section{La SRSL au sein du réseau des sociétés savantes}

Dès 1843-1844 et la parution du premier recueil de mémoires, future monnaie d'échange avec d'autres groupements savants, la SRSL apparaît toujours davantage intégrée au sein d'un réseau scientifique national et international. Constitué de sociétés d'importance et de notoriété variables, ce réseau épouse globalement les traits de la géographie (voire la géopolitique) du monde savant de ce temps. Les sociétés avec lesquelles la SRSL noue des partenariats sont tantôt, comme elle-même, des sociétés "généralistes », tantôt plus spécialisées. Comment s'opérèrent les choix a probablement souvent dépendu de contacts noués à titre individuel par tel ou tel membre de la SRSL avec un confrère belge ou étranger, membre d'une autre association. On peut par exemple penser que la présence d'Edmond de Sélys-Longchamps au sein de la Société a pesé sur l'établissement des liens avec les sociétés entomologiques apparaissant dans nos documents.

Les premiers partenaires et les plus réguliers sont belges, avec des sociétés de Bruxelles ou encore de Tournai mais surtout allemands (académies et sociétés de Münich, Berlin, Wurzburg, du Wurtemberg, de Bonn, de Stettin, du duché de Nassau, de Goerlitz, etc). Autre pôle scientifique important du temps, la France est présente via des sociétés de Bordeaux, Lille, Caen, Paris, Lyon, Montpellier, Dijon, Agen notamment.

Au-delà de ce premier cercle de partenariats, en apparaît un deuxième constitué d'académies autrichienne (Vienne, académie de Bohême), russe (Moscou et Saint-Pétersbourg), helvétique (Berne, Neuchâtel), britanniques (Londres et Dublin), hollandaise (Leyde), et du GrandDuché de Luxembourg. L'Italie est également présente, par le biais du royaume de Piémont Turin. À partir de 1853, des sociétés américaines apparaissent à leur tour dans ce paysage relationnel avec entre autres la Smithsonian Institution récemment fondée à Washington, mais aussi des sociétés établies à Philadelphie, Boston, New York, dans le Michigan, et le Wisconsin. 
Si la société liégeoise paraît d'ordinaire intéressée à l'établissement de contacts, à l'occasion et au fil du temps elle pose ses conditions et, comme elle fait en 1849 vis-à-vis du Muséum d'histoire naturelle de Paris, réclame la réciprocité pour l'envoi de ses publications. Cette attitude réservée n'est cependant pas de mise lorsqu'en 1859, elle décide de participer au capital de la future Fondation Alexandre von Humbold, à Berlin.

\section{Le revers financier de la médaille}

Ces premières années, marquées par l'expansion de la science et une croissance parallèle de la société savante liégeoise dans ce contexte favorable ne sont pourtant pas sans nuages. D'importantes difficultés financières, liées aux frais de publication de son bulletin, contrastent avec ces succès et constituent alors pour ses membres un problème lancinant. Dans le rapport annuel présenté en 1848, le secrétaire général Théodore Lacordaire évoque la "gêne » où se trouve alors la Société, même s'il tempère aussitôt ses propos :

«Il ne faut pas que nous exagérions cette gêne et y voir une cause de mort de la société. Rien n'est encore compromis, si nous montrons un peu d'énergie ».

Onze ans plus tard, en effet, il se veut plus rassurant :
" Messieurs, je ne puis que répéter ce que je vous dis chaque année (...), la société dont les premières années d'existence ont été entourées de tant de difficultés et dont l'existence a pu paraître si longtemps précaire est, si je puis m'exprimer ainsi, parvenue à l'âge viril et, à moins de circonstances imprévues, son avenir est définitivement assuré ».

Outre la vente de ses recueils, la SRSL dispose de trois sources potentielles de financement. L'une est constituée des cotisations annuelles de ses membres, (ce qui la portera parfois à souhaiter l'élargissement de son champ initial de recrutement), augmentées le cas échéant d'autres sollicitations. À partir de 1842, la SRSL réclame de même à ses adhérents une contribution extraordinaire de $10 \mathrm{fcs}$, affectée à la publication du recueil. En cas de déficit, une participation des auteurs aux frais de publication de leurs textes, au prorata de l'ampleur de ceux-ci sera également envisagée. En octobre 1845, la question financière qui se pose avec une acuité renouvelée contraint la Société à émettre un emprunt, auprès de ses membres.

Depuis sa création en 1835, l'État représente une deuxième source de revenus, sur base notamment du patronage royal, avec un subside dont le montant initial de $350 \mathrm{fcs}$, est ensuite rediscuté et réévalué.

Les besoins financiers de la Société détériorent enfin ses relations avec l'université de Liège, et son bibliothécaire Mathieu-Joseph Fiess, à l'occasion d'une controverse autour de sa bibliothèque. En quête d'argent frais, la SRSL envisage, à partir de 1849, de vendre à l'Etat pour l'université, une partie de ses livres reçus à titre d'échanges. La transaction se monterait à un montant forfaitaire de 500francs. Mais le gouvernement qui exige en contrepartie le dépôt gratuit à l'université de tous les ouvrages à recevoir par la SRSL à l'avenir crispe le débat. Le compromis qui en résulte prévoit la vente obligée à l'université des futurs livres reçus. Dans le registre des PV, cette solution se traduit par la mention à côté des titres d'une estimation de valeur. Toute source de tension n'est toutefois pas éteinte. En novembre 1850, une nouvelle contestation surgit à propos des contacts noués avec l'Académie de Dublin et de 
ses conséquences en termes de propriété d'ouvrages comme le rapporte le secrétaire Théodore Lacordaire :

\begin{abstract}
«Mr Fiess, bibliothécaire de l'université expose que c'est par suite d'une erreur que les mémoires de l'Académie de Dublin ont été adressés depuis quelques années à la Société et réclame comme appartenant à la bibliothèque qu'il dirige tout ce que la Société a reçu de ce recueil. Il est décidé qu'avant de prendre une résolution à cet égard, le Secrétaire général écrira à l'académie de Dublin pour savoir d'elle si c'est à la société ou à l'université qu'elle a entendu envoyer ses publications ».
\end{abstract}

\title{
En conclusion
}

À sa création, la SRSL bénéficie d'un contexte favorable fait de foi dans un progrès, issu du développement scientifique et technique, ainsi que de l'ancrage dans une tradition, héritée de l'Ancien Régime de sociétés savantes, impulsées par le pouvoir politique. Sitôt installée, elle s'inscrit dans le mouvement d'expansion de la science, par les travaux qu'elle promeut, comme par le réseau de partenariats qu'elle tisse à titre individuel ou institutionnel, en Belgique et au-delà de ses frontières. Sur le plan local, elle emboite résolument le pas à l'industrialisation du pays de Liège. Ces succès ne la protègent cependant pas de toute difficulté, comme le montrent ses déboires financiers.

Tels sont les enseignements du tableau fourni par les premières années de la SRSL Tableau ou plutôt esquisse, car beaucoup de questions restent à explorer dans l'histoire de cette société presque bicentenaire. Comme l'ont montré diverses histoires de sociétés savantes, si de telles associations intéressent, au premier chef, l'histoire des savoirs elles touchent aussi à l'histoire de la sociabilité, voire à celle des rapports au pouvoir.

En ce qui concerne les savoirs, il conviendrait certainement d'approfondir la question de leur contenu qui n'a été ici que superficiellement abordé. Du point de vue de la sociabilité, il y aurait de même encore beaucoup à dire. Sur les sociétés entrées en partenariat: Que sontelles, à quand remontent-elles, quel est leur rayon d'action, qui comprennent-elles dans leurs rangs ? Savoir tout cela permettrait de mieux apprécier la qualité de ces sociétés et par voie de conséquence le statut de la SRSL sur l'échelle des diverse académies.

Sur le plan des rapports au pouvoir enfin, la SRSL compte dans ses rangs de fervents catholiques comme des libéraux notoires, les deux tendances dominantes du temps, mais dans quelles proportions? Et avec quelles conséquences?

L'éventail des interrogations suscitées par ces quelques pages est vaste. Chaque piste parcourue conduit à mille autres encore à explorer. C'est ce qui, hier comme aujourd'hui, et quelle que soit la discipline abordée, fait de la recherche un métier passionnant. 